\title{
Temperature Sex Reversal Implies Sex Gene Dosage in a Reptile
}

\author{
Alexander E. Quinn, ${ }^{1 *}$ Arthur Georges, ${ }^{1}$ Stephen D. Sarre, ${ }^{1}$ Fiorenzo Guarino, ${ }^{1}$ \\ Tariq Ezaz, ${ }^{2}$ Jennifer A. Marshall Graves ${ }^{2}$
}

$\mathrm{S}$ ex is determined by genes on sex chromosomes in many vertebrates [genotypic sex determination (GSD)], but may also be determined by temperature during embryonic development [temperature-dependent sex determination (TSD)] (1). In reptiles, sex determination can involve GSD with XX and XY sex chromosomes (male heterogamety, as in mammals), GSD with ZZ and ZW sex chromosomes (female heterogamety, as in birds), or $\operatorname{TSD}(1,2)$. The distribution of TSD and GSD

A
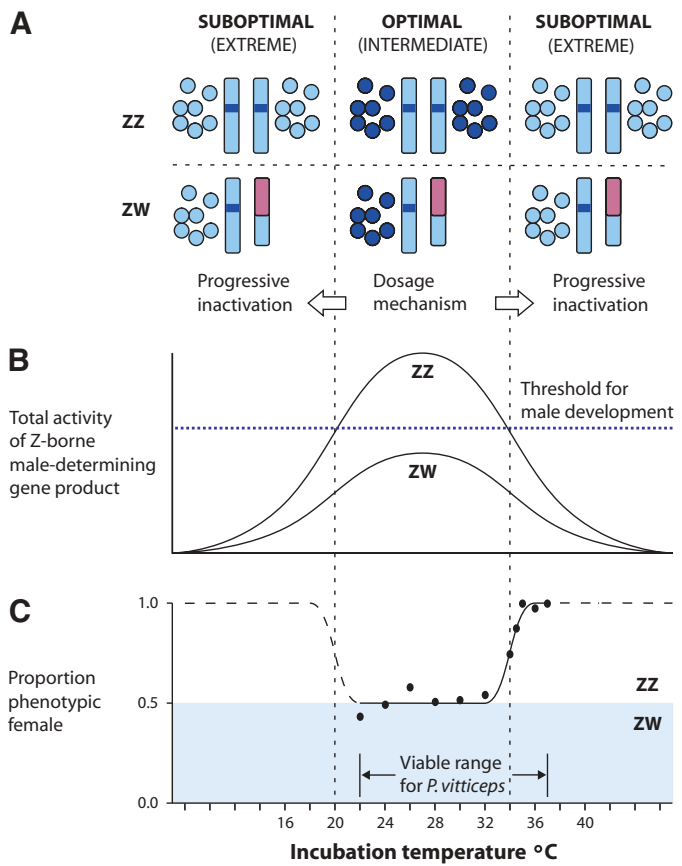

Fig. 1. Model for a ZZ dosage mechanism of sex determination in dragon lizards. (A) A Z-borne maledetermining gene (dark blue line) that expresses a temperature-sensitive product (RNA or protein) is present in two copies in ZZ individuals but in only one copy in ZW individuals. At optimal (intermediate) incubation temperatures, the gene product is fully active (dark blue circles), but it is progressively inactivated at more extreme temperatures (light blue circles). (B) In ZW individuals, the total activity of the gene product is always half that of ZZ individuals. Activity exceeds a threshold level (dashed blue line) for male differentiation only within the optimal temperature range of $\mathrm{ZZ}$ individuals. At all other temperatures, female development proceeds. (C) Thus, the phenotypic sex ratio increases from $50 \%$ females at intermediate temperatures to $100 \%$ females at temperature extremes. Data points are for $P$. vitticeps (fig. S1). across reptiles suggests several independent evolutionary transitions in sex-determining mechanisms $(2,3)$, but transitional forms have yet to be demonstrated. We show that high incubation temperatures reverse genotypic males (ZZ) to phenotypic females in the Australian central bearded dragon lizard (Pogona vitticeps), which, like birds, has GSD with female heterogamety (4). Temperature thus overrides gene(s) involved in male differentiation.

We incubated eggs of $P$. vitticeps at constant temperatures between $20^{\circ}$ and $37^{\circ} \mathrm{C}(5)$. No embryos survived to hatching at $20^{\circ} \mathrm{C}$. Between $22^{\circ}$ and $32^{\circ} \mathrm{C}$, sex ratios did not differ significantly from $1: 1$, a response consistent with GSD (fig. S1A). However, between $34^{\circ}$ and $37^{\circ} \mathrm{C}$, there was an increasing female bias, suggesting that temperature was overriding genotypic sex in some males. Differential mortality cannot explain the deviation of the sex ratio from $1: 1$ at temperatures where survivorship allowed a test (at $34.5^{\circ}, 35^{\circ}$, and $36^{\circ} \mathrm{C}$ ), because the deviation remained significant even when all mortalities were conservatively scored as male (table S1).

We isolated a female-specific DNA marker for $P$. vitticeps by screening amplified fragment length polymorphisms (AFLPs) (6) to enable a test for sex reversal (5) (fig. S1B). To verify that the AFLP marker (designated Pv72W, GenBank accession no. ED982907) is specific to the $\mathrm{W}$ chromosome, we genotyped 15 juveniles and examined their metaphase chromosome spreads. There was $100 \%$ agreement between Pv72W genotype and karyotype as demonstrated by $\mathrm{C}$ banding (11 ZW and 4 ZZ).

Pv72W was used to identify genotypic sex of hatchlings from three clutches, each split between two incubation temperatures: a control treatment $\left(28^{\circ} \mathrm{C}\right)$, which produced an unbiased phenotypic sex ratio, and a high-temperature treatment $\left(34^{\circ}\right.$ or $36^{\circ} \mathrm{C}$ ), which produced a strong female bias (table S2). Phenotypic sex was identified by hemipene eversion and examination of gonadal morphology. At $28^{\circ} \mathrm{C}$, the phenotypic sex ratio was $1: 1$, compared with 2 males and 33 females at the high temperatures. All but one of the
30 lizards $(97 \%)$ incubated at $28^{\circ} \mathrm{C}$ had concordant sex phenotype and genotype. However, only 18 of 35 animals (51\%) from the hightemperature treatment were concordant. All discordant animals were genotypic males (ZZ) that developed as phenotypic females. Our data demonstrate that high incubation temperatures reverse sex (table S2). This finding extends a previous report of low-temperature sex reversal in a skink (7) by explicitly identifying discordance between genotype and phenotype and adds empirical support for the coexistence of TSD and GSD.

The $\mathrm{W}$ chromosome is thus unnecessary for female differentiation, which suggests that the molecular mechanism directing sex determination is the dosage of a gene on the $\mathrm{Z}$ chromosome rather than the presence of a female-determining gene on the $\mathrm{W}$. That is, male differentiation requires two copies of a Z-borne gene, the expression or activity of which is sufficient for male development only at optimal temperatures (Fig. 1). We have demonstrated sex reversal at high temperatures; low-temperature sex reversal may be obscured by mortality below $22^{\circ} \mathrm{C}$.

Selection for a wider range of thermosensitivity in species such as $P$. vitticeps could result in the evolution of TSD from GSD. Reversal of the ZZ genotype to the female phenotype at extreme temperatures will bias the phenotypic sex ratio toward females and drive down the frequency of the $\mathrm{W}$ chromosome under frequency-dependent selection. This could account for the pattern observed in many TSD reptiles, where both low and high temperatures produce $100 \%$ females, yet intermediate temperatures produce predominantly (occasionally 100\%) males $(1,2)$.

\section{References and Notes}

1. ]. J. Bull, Evolution of Sex Determining Mechanisms (Benjamin/Cummings, Menlo Park, CA, 1983).

2. N. Valenzuela, V. A. Lance, Eds., Temperature-Dependent Sex Determination in Vertebrates (Smithsonian Institution, Washington, DC, 2004).

3. S. D. Sarre, A. Georges, A. Quinn, Bioessays 26, 639 (2004).

4. T. Ezaz et al., Chromosome Res. 13, 763 (2005).

5. Materials and methods are available on Science Online.

6. P. Vos et al., Nucleic Acids Res. 23, 4407 (1995).

7. R. Shine, M. J. Elphick, S. Donnellan, Ecol. Lett. 5, 486 (2002).

8. Supported by an Australian Research Council grant to S.D.S., A.G., and J.A.M.G. Experiments approved by the University of Canberra Committee for Ethics in Animal Experimentation.

\section{Supporting Online Material}

www.sciencemag.org/cgi/content/full/316/5823/411/DC1 Materials and Methods

Fig. S1

Tables $\mathrm{S} 1$ and $\mathrm{S} 2$

4 October 2006; accepted 19 January 2007 10.1126/science. 1135925

${ }^{1}$ Institute for Applied Ecology, University of Canberra, ACT 2601, Australia. ${ }^{2}$ Comparative Genomics Group, Research School of Biological Sciences, Australian National University, Canberra, ACT 0200, Australia.

${ }^{*}$ To whom correspondence should be addressed. E-mail: quinn@aerg.canberra.edu.au 